\title{
Coupled-mode theory approach to depolarization associated with propagation in turbulent media
}

\author{
B. Crosignani, P. Di Porto, and Steven F. Clifford
}

\begin{abstract}
The problem of light depolarization in a turbulent atmosphere is revisited by means of coupled-mode theory [D. Marcuse, Theory of Dielectric Optical Waveguides (Academic, New York, 1974)]. This allows, in particular, evaluation of the depolarization ratio for a plane wave and comparison of its expression with the one obtained in the frame of two distinct approaches predicting different behaviors [A. A. M. Saleh, IEEE J. Quantum Electron. QE-3, 540 (1967); J. W. Strohbehn and S. F. Clifford. IEEE Trans Antennas Propag. AP15,416 (1967)]. It is shown that both approaches give the same result when calculated to the same order in both smallness parameters relevant to the problem, thus resolving a controversy of more than 20 years.
\end{abstract}

\section{Introduction}

The analysis of depolarization induced on a light beam propagating in a turbulent medium was the object of theoretical and experimental investigation about 20 years ago. 'Since then, the subject has received little attention, also in view of the fact that depolarization effects turn out to be extremely small for the relevant case of terrestrial atmosphere. However, this topic has never been completely clarified from a theoretical point of view, since the two employed approaches ${ }^{1,2}$ produce two completely different expressions for the depolarization ratio as a function of the physical parameters of the problem (path length, wavelength, and refractive-index fluctuations). While Ref. 1 hinges on a direct generalization of the simple physical model first introduced by Hodara ${ }^{3}$ in the frame of geometrical optics, in Ref. 2 the calculations are based on the method of small perturbations. To gain deeper insight on the subject, we have reconsidered the problem by adopting a third procedure which takes advantage of coupled-mode theory, a formalism we have already employed to describe propagation of a scalar field in a turbulent medium. ${ }^{4}$ In particular, our present approach, which generalizes the scalar theory developed in Ref. 4 to include depolarization effects, turns out to provide a depolarization ratio coincident with that worked out in Ref. 1 . The apparent discrepancy between the two results in Refs. 1 and 2 can be resolved by recognizing the fact that two smallness parameters are associated with the problem and to the same order of smallness in both parameters, the results agree.

B. Crosignani is with Ugo Bordoni Foundation, 59 Via Baldassarre Castiglione, 00142 Rome, Italy; P. Di Porto is with University of L'Aquila, Physics Department, 1, P.za dell' Annunziata, 687100 L'Aquila, Italy; and S. F. Clifford is with NOAA ERL, Wave Propagation Laboratory, 325 Broadway, Boulder, Colorado 80303-3328.

Received 17 July 1987.

\section{Coupled-Mode Formalism}

Let us assume the turbulent medium to be characterized by a fluctuating refractive index of the form

$$
n(x, y, z)=n_{1}+\delta n(x, y, z) \text {, }
$$

with $|\delta n| \ll 1\left(n_{1} \cong 1\right.$ and $\delta n=10^{-6}-10^{-8}$ for terrestrial atmosphere). The coupled-mode approach consists in expanding the propagating field in terms of the modes pertaining to the ideal medium characterized by $n=n_{1}$ and to look for the evolution of the expansion coefficients as functions of the $z$-coordinate coinciding with the (average) propagation direction. This can be formalized $^{5}$ by introducing the ideal modes

$$
\begin{aligned}
& \mathbf{E}(\xi, 1 ; \mathbf{r})=N_{1} \exp (-i \xi \cdot \mathbf{r})\left[\hat{x}-\left(\xi_{x} / \beta_{\xi}\right) \hat{z}\right], \\
& \mathbf{E}(\xi, 2 ; \mathbf{r})=N_{2} \exp (-i \xi \cdot \mathbf{r})\left[\left(\xi_{x} \xi_{y} / \beta_{\xi}\right) \hat{x}-\left(\beta_{\xi}+\xi_{x}^{2} / \beta_{\xi}\right) \hat{y}+\xi_{y} \hat{z}\right],
\end{aligned}
$$

where $\mathbf{r}=(x, y), \beta_{\xi}=\left(k^{2}-\xi^{2}\right)^{1 / 2}$ with $k=2 \pi / \lambda=\omega n_{1} / c$, the transverse wave vector $\xi$ is restricted to $0<\xi<k$, and

$N_{1}=(1 / 2 \pi)\left[\beta_{\xi} \omega \mu_{0} /\left(\beta_{\xi}^{2}+\xi_{x}^{2}\right)\right]^{1 / 2}, \quad N_{2}=(1 / 2 \pi)\left[\beta_{\xi} / \omega \epsilon_{1}\left(\beta_{\xi}^{2}+\xi_{x}^{2}\right)\right]^{1 / 2}$.

The electric field is then expressed as

$$
\mathbf{E}(\mathbf{r}, z, t)=\sum_{\sigma=1}^{2} \iint d \xi \mathbf{E}(\xi, \sigma ; \mathbf{r}) c(\xi, \sigma ; z) \exp \left(i \omega t-i \beta_{\xi} z\right),
$$

where the expansion coefficients $c(\xi, \sigma, z)$ obey the set of coupled differential equations

$$
\begin{aligned}
\frac{d c}{d z}(\xi, \sigma ; z)= & \sum_{\sigma^{\prime}} \iint d \xi^{\prime}\left\{K\left(\xi, \sigma ; \xi^{\prime}, \sigma^{\prime} ; z\right)\right. \\
& \left.\times \exp \left[i\left(\beta_{\xi}-\beta_{\xi^{\prime}}\right) z\right] c\left(\xi^{\prime}, \sigma^{\prime} ; z\right)\right\},
\end{aligned}
$$

with

$$
\begin{aligned}
K\left(\xi, \sigma ; \xi^{\prime}, \sigma^{\prime} ; z\right)= & \left(\omega \epsilon_{0} / 2 i\right) \iint_{-\infty}^{+\infty} d \mathbf{r}\left[n^{2}(\mathbf{r}, z)-n_{1}^{2}\right] \mathbf{E}^{*}(\xi, \sigma ; \mathbf{r}) \\
& \cdot \mathbf{E}\left(\xi^{\prime}, \sigma^{\prime} ; \mathbf{r}\right),
\end{aligned}
$$

whose solution is equivalent to that of Maxwell's equations. From Eq. (5) we derive by means of a standard iteration procedure 


$$
\begin{aligned}
\frac{d}{d z}\left[c_{2}(\xi, 2 ; z) c_{2}^{*}\left(\xi^{\prime}, 2 ; z\right)\right]= & \int_{0}^{z} d z^{\prime} \iint d \xi^{\prime \prime} \iint d \xi^{\prime \prime \prime}\left\{\exp \left[i\left(\beta_{\xi}-\beta_{\xi^{\prime \prime}}\right) z+i\left(\beta_{\xi^{\prime \prime}}-\beta_{\xi^{\prime \prime}}\right) z^{\prime}\right]\right. \\
& \times\left[K\left(\xi, 2 ; \xi^{\prime \prime}, 2 ; z\right) K\left(\xi^{\prime \prime}, 2 ; \xi^{\prime \prime \prime}, 2 ; z^{\prime}\right) c\left(\xi^{\prime \prime \prime}, 2 ; z^{\prime}\right) c^{*}\left(\xi^{\prime}, 2 ; z^{\prime}\right)\right. \\
& +K\left(\xi, 2 ; \xi^{\prime \prime}, 2 ; z\right) K\left(\xi^{\prime \prime}, 2 ; \xi^{\prime \prime \prime}, 1 ; z^{\prime}\right) c\left(\xi^{\prime \prime \prime}, 1 ; z^{\prime}\right) c^{*}\left(\xi^{\prime}, 2 ; z^{\prime}\right) \\
& +K\left(\xi, 2 ; \xi^{\prime \prime}, 1 ; z\right) K\left(\xi^{\prime \prime}, 1 ; \xi^{\prime \prime \prime}, 1 ; z^{\prime}\right) c\left(\xi^{\prime \prime \prime}, 1 ; z^{\prime}\right) c^{*}\left(\xi^{\prime}, 2 ; z^{\prime}\right) \\
& \left.+K\left(\xi, 2 ; \xi^{\prime \prime}, 1 ; z\right) K\left(\xi^{\prime \prime}, 1 ; \xi^{\prime \prime \prime}, 2 ; z^{\prime}\right) c\left(\xi^{\prime \prime \prime}, 2 ; z^{\prime}\right) c^{*}\left(\xi^{\prime}, 2 ; z^{\prime}\right)\right] \\
& +\exp \left[i\left(\beta_{\xi}-\beta_{\xi^{\prime \prime}}\right) z-i\left(\beta_{\xi^{\prime}}-\beta_{\xi^{\prime \prime}}\right) z^{\prime}\right] \\
& \times\left[K\left(\xi, 2 ; \xi^{\prime \prime}, 1 ; z\right) K^{*}\left(\xi^{\prime}, 2 ; \xi^{\prime \prime \prime}, 2 ; z^{\prime}\right) c\left(\xi^{\prime \prime}, 2 ; z^{\prime}\right) c^{*}\left(\xi^{\prime \prime \prime}, 2 ; z^{\prime}\right)\right. \\
& +K\left(\xi, 2 ; \xi^{\prime \prime}, 2 ; z\right) K^{*}\left(\xi^{\prime}, 2 ; \xi^{\prime \prime \prime}, 2 ; z^{\prime}\right) c\left(\xi^{\prime \prime}, 2 ; z^{\prime}\right) c^{*}\left(\xi^{\prime \prime \prime}, 1 ; z^{\prime}\right) \\
& +K\left(\xi, 2 ; \xi^{\prime \prime}, 1 ; z\right) K^{*}\left(\xi^{\prime}, 2 ; \xi^{\prime \prime \prime}, 2 ; z^{\prime}\right) c\left(\xi^{\prime \prime}, 2 ; z^{\prime}\right) c^{*}\left(\xi^{\prime \prime \prime}, 2 ; z^{\prime}\right) \\
& \left.\left.+K\left(\xi, 2 ; \xi^{\prime \prime}, 1 ; z\right) K^{*}\left(\xi^{\prime}, 2 ; \xi^{\prime \prime \prime}, 1 ; z^{\prime}\right) c\left(\xi^{\prime \prime}, 1 ; z^{\prime}\right) c^{*}\left(\xi^{\prime \prime \prime}, 1 ; z^{\prime}\right)\right]\right\} \\
& +\operatorname{complex} \text { conjugate terms with the substitution} \xi \leftrightarrow \xi^{\prime}
\end{aligned}
$$

If we now assume the field to be initially polarized along the 1 direction (practically coincident with the $x$ - where we have written, according to Eq. (1), $n^{2}-n_{1}^{2} \cong$ $2 n_{1} \delta n$ and $n_{1}^{2} / n^{2} \cong 1$, so that

$$
\begin{aligned}
\left\langle K\left(\xi, 2 ; \xi^{\prime \prime}, 1 ; z^{\prime}\right) K^{*}\left(\xi^{\prime}, 2 ; \xi^{\prime \prime \prime}, 1 ; z^{\prime \prime}\right)\right\rangle= & \left(k^{2} / 4 \pi^{2} \beta_{\xi} \beta_{\xi^{\prime}}\right)\left(\xi_{x}^{\prime \prime} \xi_{y}^{\prime \prime} / \beta_{\xi^{\prime \prime}}-\xi_{x} \xi_{y}^{\prime \prime} / \beta_{\xi}\right) \\
& \times\left(\xi_{x}^{\prime \prime} \xi_{y}^{\prime \prime} / \beta_{\xi^{\prime \prime}}-\xi_{x}^{\prime} \xi_{y}^{\prime \prime} / \beta_{\xi^{\prime}}\right) \delta^{(2)}\left(\xi-\xi^{\prime \prime}+\xi^{\prime \prime \prime}-\xi^{\prime}\right) G\left(\xi^{\prime}-\xi^{\prime \prime},\left|z^{\prime}-z^{\prime \prime}\right|\right),
\end{aligned}
$$

direction) and take advantage of the small depolarization induced by the atmosphere, we can neglect on the right-hand side of Eq. (7) all the terms but the one containing $c\left(\xi^{\prime \prime}, 1 ; z^{\prime}\right) c^{*}\left(\xi^{\prime \prime \prime}, 1 ; z^{\prime}\right)$. By taking the ensemble average of the resulting equation over the possible realizations of the system, we obtain, under the hypothesis of negligible variation of $\left\langle c\left(\xi^{\prime \prime}, 1 ; z^{\prime}\right) c^{*}\left(\xi^{\prime \prime \prime}, 1 ; z^{\prime}\right)\right\rangle$ over a scale of the order of the correlation length of the refractive-index fluctuations,

$$
\begin{aligned}
\frac{d}{d z}\left\langle c(\xi, 2 ; z) c^{*}\left(\xi^{\prime}, 2 ; z\right)\right\rangle= & \int_{0}^{z} d z^{\prime} \iint d \xi^{\prime \prime} \iint d \xi^{\prime \prime \prime} \\
& \times\left\langle K\left(\xi, 2 ; \xi^{\prime \prime}, 1 ; z\right) K^{*}\left(\xi^{\prime}, 2 ; \xi^{\prime \prime \prime}, 1 ; z^{\prime}\right)\right\rangle \\
& \times\left\langle c\left(\xi^{\prime \prime}, 1 ; z^{\prime}\right) c^{*}\left(\xi^{\prime \prime \prime}, 1 ; z^{\prime}\right)\right\rangle \\
& \times \exp \left[i\left(\beta_{\xi}-\beta_{\xi^{\prime \prime}}\right) z-i\left(\beta_{\xi^{\prime}}-\beta_{\xi^{\prime \prime}}\right) z^{\prime}\right] \\
& + \text { c.c. with the substitution } \xi \leftrightarrow \xi^{\prime} .
\end{aligned}
$$

If we assume $\left\langle c\left(\xi^{\prime \prime}, 1 ; z^{\prime}\right) c^{*}\left(\xi^{\prime \prime \prime}, 1 ; z^{\prime}\right)\right\rangle$ to be given (due to small depolarization), by the expression already worked out for a linearly polarized field, ${ }^{4} \mathrm{Eq}$. (8) can be directly integrated. The amount of power transferred to the $y$-polarized component is then evaluated as ${ }^{4}$

$$
\begin{aligned}
\left\langle\left|E_{2}(\mathbf{r}, z, t)\right|^{2}\right\rangle= & \frac{1}{(2 \pi)^{2}} \iint d \xi \iint d \xi^{\prime} \exp \left\{i\left(\xi^{\prime}-\xi\right) \cdot \mathbf{r}\right. \\
& +i \beta_{\xi^{\prime}} z-i \beta_{\xi^{\prime}} z\left\langle\left\langle\mathbf{c}(\xi, 2 ; z) c^{*}\left(\xi^{\prime}, 2 ; z\right)\right\rangle .\right.
\end{aligned}
$$

\section{Evaluation of the Depolarization Ratio}

Let us first evaluate the expression of $\left\langle K\left(\xi, 2 ; \xi^{\prime \prime}, 1 ; z\right) K^{*}\left(\xi^{\prime} ; 2, \xi^{\prime \prime \prime}, 1 ; z^{\prime}\right)\right\rangle$ appearing in Eq. (8). Recalling Eq. (6), we have

$$
\begin{aligned}
K\left(\xi, 2 ; \xi^{\prime \prime}, 1 ; z\right)= & -\left(i k / 4 \pi^{2} \beta_{\xi}\right) \iint_{-\infty}^{+\infty} d \mathbf{r} \exp \left[i\left(\xi-\xi^{\prime \prime}\right) \cdot \mathbf{r}\right] \\
& \times\left(\xi_{x}^{\prime \prime} \xi_{y}^{\prime \prime} / \beta_{\xi^{\prime \prime}}-\xi_{x} \xi_{y}^{\prime \prime} / \beta_{\xi}\right) \delta n(\mathbf{r}, z),
\end{aligned}
$$

where $\delta^{(2)}$ is the 2-D delta function and

$$
\begin{aligned}
G\left(\xi^{\prime}-\xi^{\prime \prime},\left|z^{\prime}-z^{\prime \prime}\right|\right)= & \left(1 / 4 \pi^{2}\right) \iint_{-\infty}^{+\infty} d \rho \exp \left[i\left(\xi^{\prime}-\xi^{\prime \prime}\right) \cdot \rho\right] \\
& \times\left\langle\delta n\left(\rho,\left|z^{\prime}-z^{\prime \prime}\right|\right) \delta n(0,0)\right\rangle
\end{aligned}
$$

is the spatial Fourier transform of the correlation function $\left\langle\delta n(\mathbf{r}, z) \delta n\left(\mathbf{r}^{\prime}, z^{\prime}\right)\right\rangle$ of the refractive-index fluctuations associated with the turbulence field assumed to be homogeneous and isotropic.

The expression of $\left\langle c\left(\xi^{\prime \prime}, 1^{\prime} z\right) c^{*}\left(\xi^{\prime \prime \prime}, 1 ; z\right)\right\rangle$ can be found in Ref. 4 and reads for an incident plane wave

$$
\begin{aligned}
\left\langle c\left(\xi^{\prime \prime}, 1 ; z\right) c^{*}\left(\xi^{\prime \prime \prime}, 1 ; z\right)\right\rangle= & \mid E_{0}{ }^{2} \delta^{(2)}\left(\xi^{\prime \prime}-\xi^{\prime \prime \prime}\right) \pi A \\
& \times \exp \left[-A\left(\xi_{x}^{\prime 2}+\xi_{y}^{\prime 2}\right) / 4\right],
\end{aligned}
$$

with

$$
A=1 / D z,
$$

$D$ being a diffusion coefficient associated with the presence of turbulence $D=\pi^{1 / 2} k^{2}\left\langle\delta n^{2}\right\rangle / d$.

We can now substitute Eqs. (11) and (13) into Eq. (8), integrate over $z$, and insert the result into Eq. (9), thus getting

$$
\begin{aligned}
\left|E_{2}(\mathbf{r}, z, t)\right|^{2}= & \frac{1}{4 \pi} \mid E_{0}{ }^{2} k^{2} \int_{0}^{z} d z^{\prime} \int_{0}^{z} d z^{\prime \prime} \iint d \xi \iint d \xi^{\prime \prime} \\
& \times \exp \left[i\left(\beta_{\xi}-\beta_{\xi^{\prime \prime}}\right)\left(z^{\prime}-z^{\prime \prime}\right)\right] \frac{1}{\beta_{\xi} \beta_{\xi^{\prime}}} \\
& \times\left\{\frac{\xi_{x}^{\prime \prime} \xi_{y}^{\prime \prime}}{\beta_{\xi^{\prime \prime}}}-\frac{\xi_{x} \xi_{y}^{\prime \prime}}{\beta_{\xi}}\right\}\left\{\frac{\xi_{x}^{\prime \prime} \xi_{y}^{\prime \prime}}{\beta_{\xi^{\prime \prime}}}-\frac{\xi_{x} \xi_{\xi}^{\prime \prime}}{\beta_{\xi}}\right\} \\
& \times G\left(\xi-\xi^{\prime \prime}, z^{\prime}-z^{\prime \prime} \mid\right) \frac{1}{D z^{\prime \prime}} \exp \left[-\left(\xi_{x}^{\prime \prime 2}+\xi_{y}^{\prime \prime}\right) / 4 D z^{\prime \prime}\right] .
\end{aligned}
$$

Equation (15) can be approximately rewritten as 


$$
\begin{aligned}
\left|E_{2}(\mathbf{r}, z, t)\right|^{2}= & \frac{1}{4 \pi k^{2}}\left|E_{0}\right|^{2} \int d z^{\prime} \iint d \xi \iint d \xi^{\prime \prime} \xi_{y}^{\prime 2} \\
& \times\left(\xi_{x}^{\prime \prime}-\xi_{x}\right)^{2} G\left(\xi-\xi^{\prime \prime}\right) \frac{1}{D z^{\prime}} \exp \left[-\left(\xi_{x}^{\prime \prime 2}+\xi_{y}^{\prime \prime 2}\right) / 4 D z^{\prime}\right],
\end{aligned}
$$

where

$$
G\left(\xi-\xi^{\prime \prime}\right)=\int_{0}^{\infty} G\left(\xi-\xi^{\prime \prime}, \zeta\right) d \zeta,
$$

having taken advantage of the fact that $G\left(\xi^{\prime}-\xi^{\prime \prime}, \zeta\right)$ is a short-range correlation function, which goes to zero for $\zeta>d \gg \lambda$ ( $d$ being of the order of magnitude of the typical scale of turbulence) and that, for small angular divergence of the beam, $\beta \xi \cong \beta \xi^{\prime} \cong k$.

The integrals appearing in Eq. (16) can be performed after assuming a Gaussian shape for the correlation function of the refractive-index fluctuations, that is,

$$
\langle\delta n(\rho, \zeta) \delta n(0,0)\rangle=\left\langle\delta n^{2}\right\rangle \exp \left[-\left(\rho^{2}+\zeta^{2}\right) / d^{2}\right],
$$

thus getting for the depolarization ratio $R=\left\langle\left|E_{2}\right|^{2}\right\rangle /$ $\left|E_{0}\right|^{2}$ the final expression

$$
R=2 \pi\left\langle\delta n^{2}\right\rangle^{2}\left(z^{2} / d^{2}\right),
$$

which coincides with that worked out in Ref. 1.

\section{Discussion}

The dependence on $\left\langle\delta n^{2}\right\rangle, z$, and $d$ furnished by Eq. (19) is different from that worked out in Ref. 2, which reads

$$
R=4 \pi^{1 / 2}\left\langle\delta n^{2}\right\rangle(z / d)\left(1 / k^{2} d^{2}\right) .
$$

To resolve the difference between the approach that leads to Eq. (19) and that that leads to Eq. (20), we note that there are two smallness parameters, $(k d)^{-1}$ and $\delta n$, relevant to the problem. Note that the result Eq. $(19)$ is zero order in $(k d)^{-1}$ (geometric optics) and second order in $\left\langle\delta n^{2}\right\rangle$, namely, $\left\langle\delta n^{2}\right\rangle^{2}$. On the other hand, Eq. (20) is second order in $(k d)^{-1}$ and first order in $\left\langle\delta n^{2}\right\rangle$. To see if the different formulations agree we must derive the result for each to the same order of smallness in both parameters. In the Appendix we accomplish this task by extending the development of Ref. 2 to the next highest order of $\delta$, i.e., so that the variance is of the order of $\left\langle\delta n^{2}\right\rangle^{2}$ and then take the geometric optics limit, namely, to order $(k d)^{0}$. The result is Eq. (A12), which is exactly the same as Eq. (19), and the discrepancy is resolved.

The range of validity of the two approaches can be easily obtained by comparing the magnitude of the two depolarization ratios. By doing this, one can immediately check that Eq. (19) exceeds Eq. (20) for traveled distances $z$ larger than the length $z_{c}$ given by

$$
z_{c}=2\left[\sqrt{\pi}\left\langle\delta n^{2}\right\rangle k^{2} d\right]^{-1} .
$$

For typical values of the turbulence parameters $\left(\left\langle\delta n^{2}\right\rangle=10^{-14}\right.$ and $d$ of the order of a meter), $z_{c}$ turns out to be, at optical frequencies $\left(k=10^{5} \mathrm{~cm}^{-1}\right)$, of the order of a few meters, while, at millimeter wavelengths $\left(k=10^{8} \mathrm{~cm}^{-1}\right), z_{c}$ is of the order of thousands of kilometers.

\section{v. Conclusions}

We have developed a formalism, which, as a generalization of a previous one based on coupled-mode theory and dealing with scalar propagation in a turbulent medium, allows us to describe vector propagation. In particular, our method is employed to evaluate the depolarization undergone by an initially linearly polarized plane wave and to compare the corresponding depolarization ratio with the results worked out in the frame of other approaches. Also, if the depolarization effects are in practice very small, this comparison turns out to be very useful for checking the range of validity of the different approaches, respectively, based on methods of small perturbations and coupled-mode theory. We show, for the first time, that both formalisms give identical results when calculated to the same order in the two smallness parameters [ $\delta n$ and $\left.(k d)^{-1}\right]$ relevant to the problem. This resolves a discrepancy that has existed in the literature for more than twenty years.

This work has been partially supported by Ministero della Pubblica Istruzione.

This paper was presented at the May 1987 Agard Meeting on Scattering and Propagation in Random Media, Rome, Italy.

\section{Appendix}

To resolve the differences between Eqs. (19) and (20), we note that the former equation is zero order in $(k d)^{-1}$ and is second order in refractive-index variance $\left\langle\delta n^{2}\right\rangle^{2}$, whereas the latter equation is first order in $\left\langle\delta n^{2}\right\rangle$ but is second order in $(k d)^{-1}$. The strategy here is to use the technique of Ref. 2 to obtain the equivalent result for $R$ that is both zero order in $(k d)^{-1}$ and second order in $\left\langle\delta n^{2}\right\rangle$. Then, if physics prevails, the result should be identical to Eq. (19), and no discrepancies among the methods will exist.

To begin, we note that in Ref. 2, Strohbehn and Clifford retained terms in the electric field fluctuations to first order only in $\delta n$. As a consequence, they could not have obtained the result Eq. (19) and indeed, correctly obtained, $R=0$ to first order in $\left\langle\delta n^{2}\right\rangle$ in the geometric optics limit as $(k d)^{-1} \rightarrow 0$. However, we now wish to compare results so we retain terms to second order in $\delta n$ in their governing equations. Starting with Eq. (1) of Ref. 2 and retaining terms to second order in $\delta n$, we obtain

$$
\begin{gathered}
\nabla^{2} \mathbf{E}_{2}+k^{2} \mathbf{E}_{2}+2 k^{2} \delta n \mathbf{E}_{1}+k^{2} \delta n^{2} \mathbf{E}_{0}+ \\
2 \nabla\left[\mathbf{E}_{1} \cdot \nabla(\delta n)\right]-\nabla\left[\mathbf{E}_{0} \cdot \nabla\left(\delta n^{2}\right)\right]=0,
\end{gathered}
$$

where $\mathbf{E}(x, y, z)=\mathbf{E}_{0}+\mathbf{E}_{1}+\mathbf{E}_{2}+\ldots$ is a perturbation series in the electric field vector with successively higher-order terms, $\mathbf{E}_{m}$ is of the order of smallness $(\delta n)^{m}$. As before, we assume an initial plane wave polarized in the $x$ direction, propagating in the $z$ direction, and calculate the depolarized field $E_{2 y}$. (Note that to second order in $\delta n, \mathbf{E}_{y}=\mathbf{E}_{2 y}$ when we go to the geometric optics limit.) If we retain only those source terms for 
$E_{2 y}$ that survive in the limit $(k d)^{-1} \rightarrow 0$, Eq. (A1) reduces to

$$
\nabla^{2} E_{2 y}+k^{2} E_{2 y}=-2 k^{2} \delta n E_{1 y},
$$

which has the solution

$$
E_{2 y}(\mathbf{r})=-\frac{1}{4 \pi} \int\left[-2 k^{2} \delta n\left(\mathbf{r}^{\prime}\right) E_{1 y}\left(\mathbf{r}^{\prime}\right)\right] \frac{\exp (i k \mid \mathbf{r}-\mathbf{r} 1)}{|\mathbf{r}-\mathbf{r}|} d^{3} \mathbf{r}^{\prime},
$$

where $\mathbf{r}$ is a vector from the origin of coordinates to the observation point, and $\mathbf{r}^{\prime}=\left(\rho^{\prime}, x^{\prime}\right)$ describes the scattering volume. From Ref. 2 we can write the expression for the depolarization ratio $M$ in the form

$$
\begin{aligned}
M_{1}\left(\rho^{\prime}, z^{\prime}\right)= & \frac{E_{1 y}\left(\mathbf{r}^{\prime}\right)}{E_{0}\left(\mathbf{r}^{\prime}\right)}=-\frac{i}{k} \int \exp \left(i \mathbf{K} \cdot \rho^{\prime}\right) K_{x} K_{y} \int_{0}^{z^{\prime}} d z^{\prime \prime} \\
& \times \exp \left[-i \frac{K^{2}\left(z^{\prime}-z^{\prime \prime}\right)}{2 k}\right] d \nu\left(\mathbf{K}, z^{\prime \prime}\right),
\end{aligned}
$$

where we have introduced the Fourier-Stieltjes measure $d \nu\left(\kappa^{\prime}, z^{\prime}\right)$ according to the form

$$
\delta n\left(\boldsymbol{\rho}^{\prime}, z^{\prime}\right)=\int \exp \left(i \mathbf{K} \cdot \boldsymbol{\rho}^{\prime}\right) d v\left(\mathbf{K}, z^{\prime}\right),
$$

where $\mathbf{K}=\left(K_{x}, K_{y}\right)$.

Inserting Eq. (A4) into Eq. (A3), we can make the parabolic approximation and write the expression for the on-axis depolarization ratio in the form

$$
\begin{aligned}
M_{2}(0, z)= & \frac{E_{2 y}(0, z)}{E_{0}(0, z)} \\
= & -\frac{i k}{2 \pi} \int_{0}^{z} \frac{d z^{\prime}}{\left(z-z^{\prime}\right)} \int_{0}^{z^{\prime}} d z^{\prime \prime} d \nu\left(\mathrm{K}, z^{\prime \prime}\right) \int K_{y} K_{x} \\
& \times \exp \left[-\frac{i K^{2}\left(z^{\prime}-z^{\prime \prime}\right)}{2 k}\right] \int d^{2} \rho^{\prime} \\
& \times \exp \left[\frac{i k \rho^{\prime 2}}{2\left(z-z^{\prime}\right)}+i \mathrm{~K} \cdot \rho^{\prime}\right] \delta n\left(\rho^{\prime}, z^{\prime}\right) .
\end{aligned}
$$

Again making use of Eq. (A5) and dropping the Fresnel terms, which is allowable in the geometric optics limit, we obtain

$$
M_{2}(0, z)=\int_{0}^{z} d z^{\prime} \int_{0}^{z^{\prime}} d z^{\prime \prime} \int K_{y} K_{x} d \nu\left(\mathbf{K}, z^{\prime \prime}\right) \int d \nu\left(\mathbf{K}^{\prime}, z^{\prime}\right)
$$

We now compute the variance

$$
\begin{aligned}
R= & \left\langle M_{2}^{2}(0, z)\right\rangle=\int_{0}^{z} d z_{1}^{\prime} \int_{0}^{z_{1}^{\prime}} d z_{1}^{\prime \prime} \int_{0}^{z} d z_{2}^{\prime} \\
& \times \int_{0}^{z_{2}^{\prime}} d z_{2}^{\prime \prime} \iint K_{1 y} K_{1 x} K_{2 y} K_{2 x} \\
& \times \iint\left\langle d \nu\left(\mathbf{K}_{1}, z_{1}^{\prime \prime}\right) d \nu\left(\mathbf{K}^{\prime}, z_{1}^{\prime}\right)\right. \\
& \left.\times d \nu^{*}\left(\mathbf{K}_{2}, z_{2}^{\prime \prime}\right) d \nu^{*}\left(\mathbf{K}^{\prime \prime}, z_{2}^{\prime}\right)\right\rangle .
\end{aligned}
$$

We can simplify Eq. (A8) drastically by employing the Markov approximation, ${ }^{6}$ which for a Gaussian random process $d \nu$ implies

$$
\begin{aligned}
\left\langle d \nu d \nu d \nu^{*} d v^{*}\right\rangle= & (2 \pi)^{2} \delta\left(\mathbf{K}_{1}-\mathbf{K}_{2}\right) \delta\left(z_{1}^{\prime \prime}-z_{2}^{\prime \prime}\right) \Phi_{n}\left(\mathbf{K}_{1}\right) d^{2} \mathbf{K}_{1} \cdot d^{2} \mathbf{K}_{2} \\
& \times \delta\left(\mathbf{K}^{\prime}-\mathbf{K}^{\prime \prime}\right) \delta\left(z_{1}^{\prime}-z_{2}^{\prime}\right) \Phi_{n}\left(\mathbf{K}^{\prime}\right) d^{2} \mathbf{K}^{\prime} d^{2} \mathbf{K}^{\prime \prime} \\
& +(2 \pi)^{2} \delta\left(\mathbf{K}^{\prime}-\mathbf{K}_{2}\right) \delta\left(z_{1}^{\prime}-z_{2}^{\prime \prime}\right) \Phi_{n}\left(\mathbf{K}^{\prime}\right) \\
& \times d^{2} \mathbf{K}^{\prime} d^{2} \mathbf{K}_{2} \delta\left(\mathbf{K}_{1}-\mathbf{K}^{\prime \prime}\right) \delta\left(z_{1}^{\prime \prime}-z_{2}^{\prime}\right) \\
& \times \Phi_{n}\left(\mathbf{K}_{1}\right) d^{2} \mathbf{K}_{1} d^{2} \mathbf{K}^{\prime \prime} \\
& +(2 \pi)^{2} \delta\left(\mathbf{K}_{1}+\mathbf{K}^{\prime}\right) \delta\left(z_{1}^{\prime \prime}-z_{1}^{\prime}\right) \Phi_{n}\left(\mathbf{K}_{1}\right) \\
& \times d^{2} \mathbf{K}_{1} d^{2} \mathbf{K}^{\prime} \delta\left(\mathbf{K}_{2}+\mathbf{K}^{\prime \prime}\right) \delta\left(z_{2}^{\prime \prime}-z_{2}^{\prime}\right) \\
& \times \Phi_{n}\left(\mathbf{K}_{2}\right) d^{2} \mathbf{K}_{2} d^{2} \mathbf{K}^{\prime \prime}
\end{aligned}
$$

After Eq. (A9) is substituted into Eq. (A8) only the first term will contribute. The other terms will drop out because of the oddness of the integrand over $K_{y}$ and $K_{x}$. After substituting and integrating over the delta functions, we have

$$
\begin{aligned}
R= & (2 \pi)^{2} \int_{0}^{z} d z_{1}^{\prime} z_{1}^{\prime} \int d^{2} \mathbf{K}_{1} K_{1 x}^{2} K_{1 y}^{2} \\
& \times \Phi_{n}\left(\mathbf{K}_{1}\right) \int d^{2} \mathbf{K}^{\prime} \Phi_{n}\left(\mathbf{K}^{\prime}\right) .
\end{aligned}
$$

To complete the calculation we assume the covariance function for $\delta n$ given by Eq. (18) in the text, which implies that

$$
\begin{aligned}
\Phi_{n}(\mathbf{K}) & \equiv \frac{1}{(2 \pi)^{3}} \int d^{3} \mathbf{r}\langle\delta n(\mathbf{r}) \delta n(0)\rangle \exp (i \mathbf{K} \cdot \mathbf{r}) \\
& =\frac{d^{3}\left\langle\delta n^{2}\right\rangle}{8 \pi^{3 / 2}} \exp \left(-K^{2} d^{2} / 4\right) .
\end{aligned}
$$

Inserting Eq. (A11) into Eq. (A10), we finally obtain

$$
R=2 \pi\left\langle\delta n^{2}\right\rangle^{2}\left(z^{2} / d^{2}\right)
$$

or exactly the result of Eq. (19) in the text, which was to be proved.

\section{References}

1. A. A. M. Saleh, "An Investigation of Laser Wave Depolarization Due To Atmospheric Transmission," IEEE J.Quantum Electron. QE-3, 540 (1967).

2. J. W. Strohbehn and S. F. Clifford, "Polarization and Angle-ofArrival Fluctuations for a Plane Wave Propagated Through a Turbulent Medium," IEEE Trans. Antennas Propag. AP-15, 416 (1967).

3. H. Hodara, "Laser Wave Propagation Through the Atmosphere," Proc. IEEE 54, 368 (1966).

4. B. Crosignani and P. Di Porto, "Electromagnetic Propagation in a Turbulent Medium: a New Approach," J. Opt. Soc. Am. 73, 1581 (1983).

5. B. Crosignani and A. Yariv, "Degenerate Four-Wave Mixing in the Presence of Nonuniform Pump Wave Fronts," J. Opt. Soc. Am. A 1, 1034 (1984).

6. J. W. Strohbehn, Ed., Laser Beam Propagation in the Atmosphere (Springer-Verlag, New York, 1978). 